\title{
Assessing the effect of the electrode orientation on the performance of soil microbial fuel cells
}

\author{
Jakub Dziegielowski ${ }^{1,2}$, Gresa Bregu ${ }^{1}$, Luke Hulse ${ }^{1}$ and Mirella Di Lorenzo ${ }^{1,2, *}$ \\ ${ }^{1}$ Department of Chemical Engineering, University of Bath, BA2 7AY, Bath, United Kingdom \\ ${ }^{2}$ Centre for Biosensors, Bioelectronics and Biodevices, University of Bath, BA2 7AY, Bath, United Kingdom
}

\begin{abstract}
Soil microbial fuel cells (SMFCs) are a sub-class of the microbial fuel cells family, in which the soil acts as the electrolyte, and as the source of microorganisms and organic fuel. Given the great simplicity of the system design, SMFCs show a promising avenue for energy generation in remote areas. In this study, we investigate the influence that geometrical factors, such as the electrode orientation, have on the electrochemical performance of SMFCs. Two types of electrode orientations: horizontal and vertical, were tested. Additionally, the influence of anode and cathode immersion in soil was explored too. Our results demonstrate that vertical positioning of the cathode in soil is not a viable option. The increase in cathodic immersion leads to a more rapid performance decay, attributed to more anaerobic conditions along soil's depth. The increase in anode immersion has a positive effect on the evolution of the negative electrode potential. However, with the increase in electrode spacing, the performance drops due to a greater internal resistance.
\end{abstract}

\section{Introduction}

The threat of climate change pushes the search for alternative energy sources that minimize carbon pollution. In this context, Microbial Fuel Cells hold great promises. Particularly interesting are Soil Microbial Fuel Cells (SMFCs), capable of deriving electricity from soil, via microbial and electrochemical mechanisms. In these systems, the soil acts as the electrolyte and as the source of both microorganisms and organic matter. With respect to other types of microbial fuel cells, SMFCs are characterized by an extremely simple and cost-effective design, with no need for fuel pumping and/or electrolyte replacement. Minimum maintenance is required since the organic matter is naturally provided in the soil by plants and/or decomposing matter, thus allowing long term operations in remote areas.

Yet, the feasibility of this technology for real life applications is often questioned, due to the low power output, which is a consequence of the inherent thermodynamic limitations. In microbial fuel cells, the electromotive force induced by the bioelectrochemical reactions is limited to a theoretical maximum potential of $1.14 \mathrm{~V}$, resulting in power densities in the range of tens to hundreds of $\mathrm{mW} \mathrm{m}^{-2}$ [1]. Redox kinetics is a barrier that may never be fully broken through, despite many great attempts: implementing oxygen reduction reaction catalysts at the cathode [2]; integrating membranes and gas diffusion layers [3]; selecting specific microbial strains or genetically modifying them for optimum performance [4]. Consequently, alternative approaches are necessary to try and maximise power densities.

Engineering a space efficient device could be a great breakthrough in commercialising SMFCs, since the power scale-up can only be achieve by stacking together several SMFC units [5]. The surface coverage required by a single SMFC is therefore a current challenge, due to the necessity for cathode's exposure to air [5]. Wetser et al attempted to resolve this, by developing a tubular reactor, operational underground [6]. The device, however, required pure oxygen or pressurised air to be pumped through, resulting in higher energy requirements. It also suffered from instability due to oxygen crossover from cathode to anode, which significantly lowered the electric output [6]. Walter et al showed a promising concept by submerging vertically positioned electrodes in urine fed microbial fuel cells [7]. They found the performance improving with increased immersion of the device in urine, (with optimum immersion of $75 \%$ ), due to enhancements in reduction reaction rates from better contact between the cathode and the electrolyte [7].

Therefore, to test whether a similar approach can be used in soil microbial fuel cells, in this study, we investigate the effect that positioning the electrodes vertically, rather than horizontally, has on electrochemical performance. This geometrical approach has the potential to enhance output power densities, by allowing more SMFCs installed per surface area.

\footnotetext{
* Corresponding author: m.di.lorenzo@bath.ac.uk
} 


\section{Materials and Methods}

\subsection{Materials}

All reagents used were purchased of analytical grade from Alfa Aesar and Sigma-Aldrich, unless otherwise specified, and used without further purification. The soil was collected from a maximum depth of $30 \mathrm{~cm}$ below the surface around the campus area of the University of Bath. Soil pH $(6.44 \pm 0.19)$ and conductivity $(\kappa=195.2$ $\pm 23.3 \mu \mathrm{S} \mathrm{cm}^{-1}$ ) were measured by using a Thermo Scientific Orion Star A325 probe. The moisture content expressed as a percentage by mass, Wm $(\% \mathrm{~m} / \mathrm{m})$, calculated according to Eq. 1, was $45.05 \pm 0.36 \%$.

$$
W_{m}=\frac{m_{w}-m_{D}}{m_{w}-m_{0}} \times 100
$$

where $\mathrm{mw}(\mathrm{g})$ is the mass of the wet sample in the crucible; $\mathrm{mD}(\mathrm{g})$ is the mass of the dried sample in the crucible; $\mathrm{m} 0(\mathrm{~g})$ is the mass of the empty crucible. The organic matter content, $21.75 \pm 0.47 \%$ (expressed as a percentage by mass) was estimated with the Loss on Ignition analysis.

\subsection{SMFC design and operation}

Six membrane-less air-cathode SMFC designs were constructed. In three of the devices, the electrodes were configured vertically, while in the other three, a horizontal electrode orientation was used. Each design had three replicates to evaluate the reliability and reproducibility of the data obtained. The design specifications of the six SMFCs used in this study are summarised in Table 1.

Table 1. SMFCs tested in the study

\begin{tabular}{cccc}
\hline $\begin{array}{c}\text { SMFC } \\
\text { Design }\end{array}$ & $\begin{array}{c}\text { Electrode } \\
\text { Configuration }\end{array}$ & $\begin{array}{c}\text { Electrode } \\
\text { Spacing }\end{array}$ & $\begin{array}{c}\text { Cathode soil } \\
\text { immersion }\end{array}$ \\
\hline H-4 & Horizontal & $4 \mathrm{~cm}$ & - \\
H-6 & Horizontal & $6 \mathrm{~cm}$ & - \\
H-8 & Horizontal & $8 \mathrm{~cm}$ & - \\
V-25 & Vertical & $2 \mathrm{~cm}$ & $25 \%(2 \mathrm{~cm})$ \\
V-50 & Vertical & $2 \mathrm{~cm}$ & $50 \%(4 \mathrm{~cm})$ \\
V-75 & Vertical & $2 \mathrm{~cm}$ & $75 \%(6 \mathrm{~cm})$ \\
\hline
\end{tabular}

The SMFC devices were fit into PVC containers $(32 \times 24 \times 10 \mathrm{~cm})$ filled up with soil, which was kept moisturised with tap water. The anodes were buried inside the soil and the cathodes were exposed to air. In the case of the vertical designs, cathode immersion in soil varied, as shown in Figure 1. The electrodes were connected to an external resistance of $510 \Omega$ and to a data acquisition system (DAQ6510, Keithley), by means of titanium wire $(0.25 \mathrm{~mm}$ diameter $)$. Polarization tests were performed by connecting the SMFCs to a resistance box (Cropico RM6 Decade) and varying the applied resistance. Ohm's law $\left(\mathrm{I}=\mathrm{E} / \mathrm{R}_{\mathrm{ext}}\right)$ was used to calculate the current (I) corresponding to each externa resistance $\left(\mathrm{R}_{\text {ext }}\right)$.

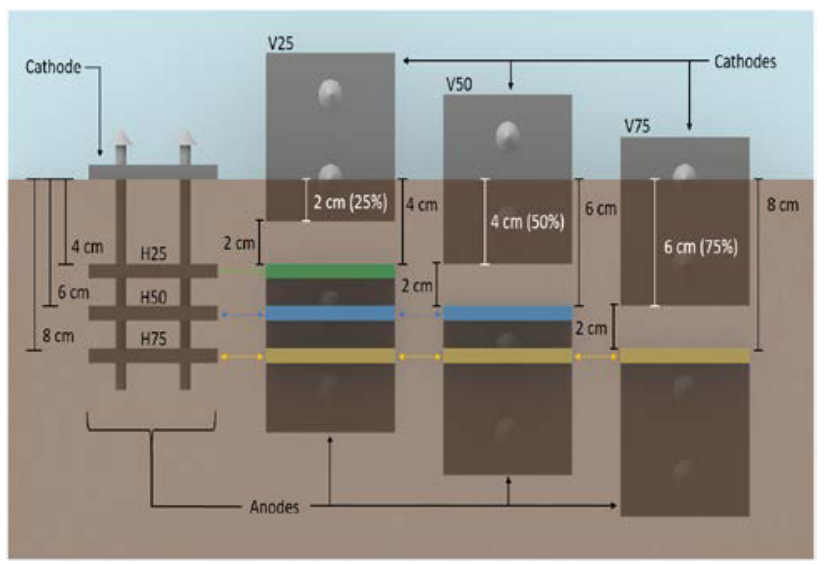

Fig.1. SMFC designs tested in this study.

\section{Results}

The performance of the several SMFCs was assessed and compared according to the enrichment curves (Figure 2). As shown in Fig.2A, for all the SMFCs with horizontal electrodes the lag phase took 9.65 days for $\mathrm{H}-8$, followed by 10.76 days for $\mathrm{H}-6$ and 11.41 days for $\mathrm{H}-4$. The SMFCs with vertical electrodes (Fig.2B) experienced an average of $5 \%$ increase in microbial growth rate, and $42 \%$ decrease in lag phase duration, with V-25 (7.26 days), V-50 (6.3 days) and V-75 (4.86 days), respectively. These variances were found to be related to the anode depth and orientation. Evaluation of individual electrode potentials revealed that the reactor designs with the vertical anodes immersed deeper in the soil, reach negative values of $-400 \mathrm{mV}$ vs. $\mathrm{Ag} / \mathrm{AgCl}$, six days faster than the horizontal set-ups (see Figure 3).
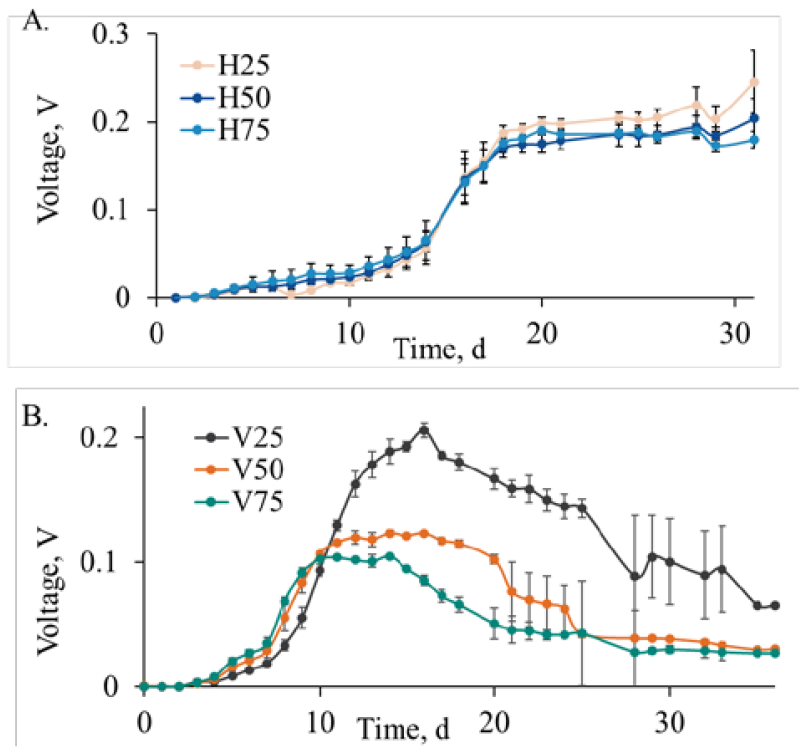

Fig.2. Enrichment curves of: (A) Horizontal reactor designs; (B) Vertical reactor designs. Data is the average of three replicates. Error bars refer to 3 replicates. 
This result can be attributed to the increased anaerobic conditions, at the anode in deep soil [8], where a more rapid electroactive biofilm development allows the exponential growth phase to be reached quicker.

The other noticeable difference between the designs is in the steady-state voltage. The horizontal configuration produced a stable steady voltage for the entire duration of the experiment (Fig. 2A), whereas the voltage of the vertical configurations started to decay after only two weeks of operation (Fig. 2B). Although the exact reason for this voltage decay was not identified, individual electrode potential assessment revealed that the drop in performance is related to the cathodes. As shown in Fig. 3, following the enrichment completion, the cathode potentials in all the vertical configurations began to decrease from $-0.27 \pm 0.05 \mathrm{~V}$ to $-0.41 \pm 0.02 \mathrm{~V}$, after 15 days.
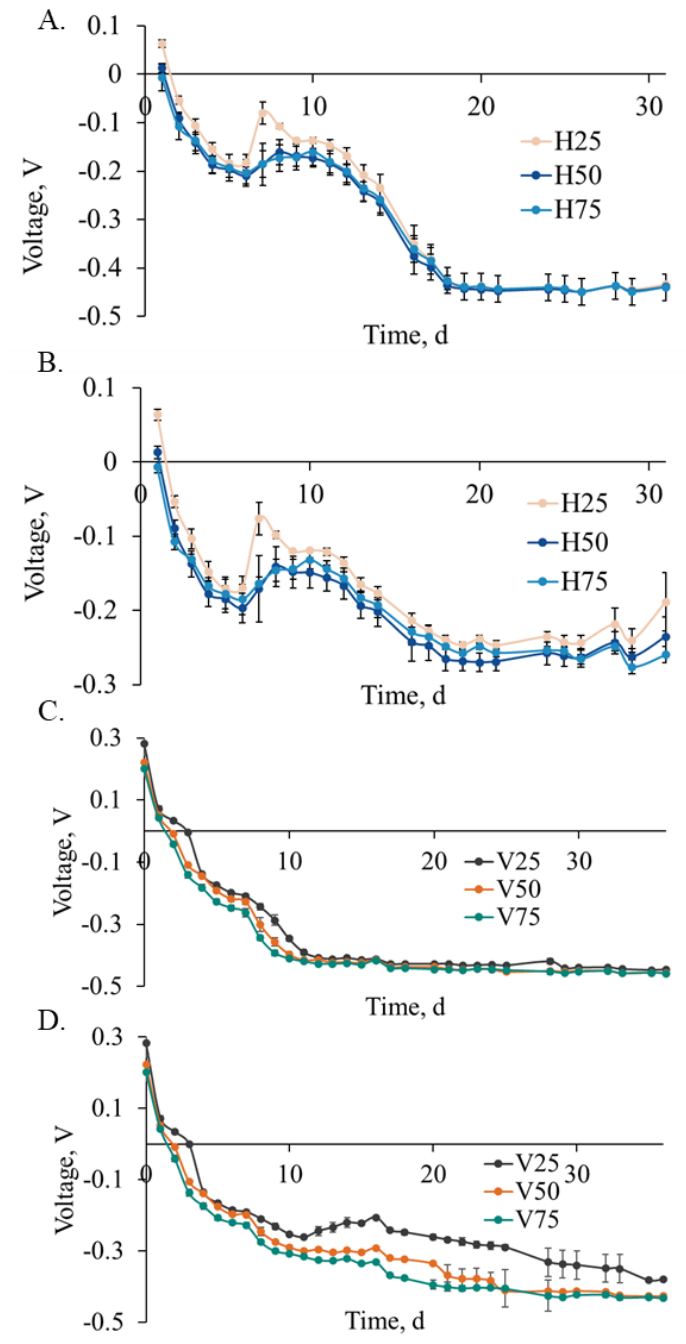

Fig.3. (A) Anode potentials of Horizontal SMFCs; (B) Cathode potentials of Horizontal SMFCs; (C) Anode potentials of Vertical SMFCs; (D) Cathode potentials of Vertical SMFCs

This voltage decay suggests increasing oxidative conditions at the cathode. A quantitative assessment of the growth curves revealed that the rate of voltage decay decreased with time. In particular, the output voltage generated by V50 and V75 dropped by $15.62 \%$ and $21.36 \%$, respectively, compared to V25.
This result, therefore, confirms that the greater the cathode fraction immersed in soil, the higher the output voltage decay.

The voltage versus time trends obtained can be attributed to the increasingly anoxic conditions experienced at greater soil depths, which can decrease the rate of reduction reaction, due to oxygen limitations [9]. Furthermore, it is likely that a mix potential distribution occurs within the electrodes partially immersed in the soil, to the point that the part of the electrode deeper in the soil may act as the anode and transfer electrons to the upper part of the electrode, thus short circuiting the overall system [10].

Further analysis was carried out in the form of polarisation tests, which were done on day 20 , after completion of the enrichment, and on day 30, following the voltage decay of vertical SMFCs. In the case of the SMFCs with horizontal electrodes, the configuration with a $4 \mathrm{~cm}$ electrode spacing performed best, generating $0.110 \mathrm{~mW}$. Second was the configuration with a $6 \mathrm{~cm}$ spacing $(0.082 \mathrm{~mW})$, and third the configuration with a 8 $\mathrm{cm}$ spacing $(0.079 \mathrm{~mW})$. Furthermore, the performance of the H-25 and H-50 configurations increased from day 20 to day 30 by $68 \%$ and $32.5 \%$, respectively, which can be attributed to extra developments of electroactive biofilms [11]. The performance of H-75 slightly dropped by $13 \%$ after the 10 days. This trend suggests that $4 \mathrm{~cm}$ is the optimal distance between the electrodes in a flatplate configuration. Distances smaller than $4 \mathrm{~cm}$ can lead to oxygen crossover in the anode [12] and distances larger than $4 \mathrm{~cm}$ begin to inhibit proton diffusion, causing greater internal resistances, as demonstrated in Figure 4A.
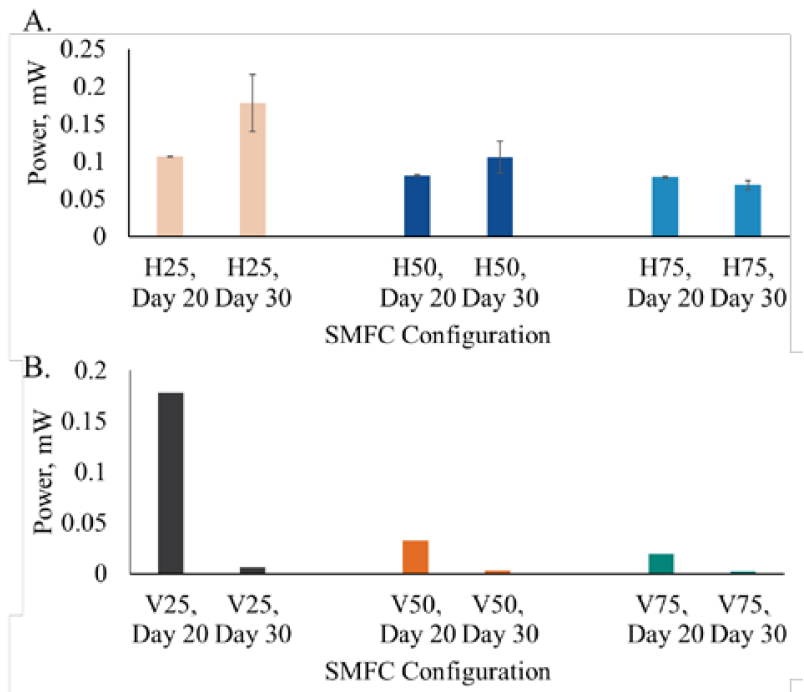

Fig.4. Maximum power generated by the SMFCs after 20 and 30 days of operation; (A) Horizontal set-up; (B) Vertical set-up

In the case of vertical SMFCs (Fig.4B), on day 20, V-25 significantly outperformed the other configurations, generating $0.178 \mathrm{~mW}$. However, as a consequence of the voltage decay, the performance in all vertical SMFCs dropped. V-25, V-50 and V-75 generated only $0.006 \mathrm{~mW}, 0.003 \mathrm{~mW}$ and $0.002 \mathrm{~mW}$, respectively, after 30 days of operation. 


\section{Conclusions}

Soil microbial fuel cells have tremendous potential in energy harvesting applications in remote areas. In this study, the effect of the electrode orientation was investigated to identify solutions that would enhance the power density of SMFCs stacks. SMFCs with horizontal and vertical electrical configurations were tested. The tests revealed that the electrode configuration and placement in soil has clearly an effect on performance in SMFCs. Cathode immersion showed a negative influence, causing an up to $68 \%$ voltage decay after four weeks of operation. Although the exact reason for this behaviour has not been identified, it is likely related to slower reduction rates due to oxygen limitations across the depth of soil and mixed potentials developing along the electrode. It was also demonstrated that the optimal spacing between horizontal electrodes is $4 \mathrm{~cm}$, generating $58 \%$ and $158 \%$ more power than reactors with $6 \mathrm{~cm}$ and $8 \mathrm{~cm}$ spacing, resulting in power densities of $28 \mathrm{mWm}^{-2}$. Although low, such power would be able to sustain the operation of certain electronics and LEDs, or could be accumulated and stored over, to power devices with higher energy requirements.

This project was funded by the UK Research England, under the Global Challenges Research Fund (GCFR), and is part of the project GREENER funded by the European Union's Horizon 2020 research and innovation programme under the grant agreement No 826312

\section{References}

[1] C. Santoro, C. Arbizzani, B. Erable, I. Ieropoulos., Power Sources 356 (2017), pp. 225-244

[2] H. Yuan, Y. Hou, I. Abu-Reesh, J. Chen, Z. He, Mater. Horiz, 3, (2016), pp. 382-401

[3] M. Shabani, H. Younesi, M. Pontie, A. Rahimour, M. Rahimejad, A. Zinatizadeh, J. Clean. Prod., 264, (2020)

[4] M. Angelaalincy, R. Krishnaraj, G. Shakambari, B. Ashokkumar, S. Kathiresan, P. Varalakshmi, Front. Energy Res., (2018)

[5] J. Dziegielowski, B. Metcalfe, P. Guzman, C.A. Martinez-Huitle, A. Gotrayeb, J. Wenk, M. Di Lorenzo, Appl Energy, 278 (2020)

[6] K. Wetser, K. Dieleman, C. Buisman, D. Strik, Appl Energy, 185 (2017), pp.642-649

[7] X. Waletr, C. Santoro, J. Greenman, I. Ieropoulos, Int. J. Hydrog. Energy, 44 (2019), pp. 4524-4532
[8] Chen, C.-Y., et al., 2013. Environmental Technology 35, 2013, p. 286-293.

[9] H. Rismani-Yazdi, et al., J Power Sources, 180, (2008), pp. 683-694.

[10] F. Harnisch, U. Schroder, ChemSusChem, 2, (2009), pp. 921-926.

[11] G. Pasternak, J. Greenman, I. Ieropoulos, J Power Sources, 400, (2018), pp. 392-401

[12] J. Dziegielowski, N. Poyiatzi, M. Di Lorenzo, EFC19 (2019) 